\author{
OBSERVATIONAL TECHNIQUES AND \\ RESULTS FOR VISUAL DOUBLE STARS \\ by \\ Charles E. Worley \\ U.S. Naval Observatory, Washington, D.C.
}

Introduction

A little more than a century and a half has passed since F.G.'. Struve began to provide the astronomical world with visual double star measures of both quantity and quality. Since that time, nearly a million individual measures have been made by all techniques, and most of these are now available in machinereadable form in the punch-card Observation Catalog maintained at the Naval Observatory. As of 1 March 1981, this Catalog contained 877242 measures grouped into 398818 means. The data file is now virtually complete for all 20 th century observations, and perhaps $80 \%$ complete for the 19 th century material. Additions are being made steadily and we can look forward to having a complete set of observations for every known double star in a few more years.

Table 1 shows the distribution of the means according to observational technique. Note that well over $80 \%$ of all the positional data we have for visual binaries has been contributed by visual observers using one form or another of micrometer. If we examine the data for the close pairs, and in particular those objects showing enough orbital motion so that an orbit has been deduced, the contribution of the visual technique would rise to over $95 \%$ of the usable observations. It is just this body of data, of course, combined with accurate parallaxes and mass-ratios, which defines the mass-luminosity relation for the stars of spectral type $A$ and later.

Table 1.

Data Obtained for Visual Double Stars by Different Techniques

Method

Visual:

Photographic:

Interferometric:

with filar micrometer with double-image micrometer with long-focus telescopes with short-focus telescopes visually speckle
No. Means

$$
\begin{array}{rr}
323, & 000 \\
7, & 000 \\
30, & 000 \\
29, & 000 \\
7, & 000 \\
1, & 000 \\
2, & 000
\end{array}
$$

Tota1 399,000 


\section{Visual Techniques}

Many different types of instruments have been developed to measure binaries visually. However, it is safe to say that the filar micrometer, in use since the time of F.G.W. Struve, remains the paramount device for this work. I will return to a discussion of the filar micrometer after first giving consideration to some other significant instruments.

Hargreaves (1932) and Davidson and Symms (1938) designed and put into operation at the Greenwich Observatory a comparison-image micrometer, in which an artificial double star of appropriate magnitude and color was projected into the focal plane. Because no wire illumination was necessary, it was claimed that fainter stars could be measured than would have been possible with the filar micrometer: in fact, the bright London sky precluded observations of faint pairs anyway. Greater accuracy in measuring separations was also claimed, particularly for inexperienced observers, and the later Greenwich observations do seem better than those made before, say, 1930, although they are not really superior to those made by the conventional filar micrometer. Instruments bearing some resemblance to the comparison-image micrometer have also been used by Duruy (1938) and Camichel (1956), but only small numbers of measures have been made with them.

There are numerous kinds of double-image micrometers. In fact, the heliometer represents an early example of such an instrument, and has been used to make a few double star measures. Unfortunately, the high magnifying powers necessary for this work emphasize the serious aberrations of the split lens, and the measures are not very useful. A better scheme was devised by Amici (1815) and Airy (1846) in which the eyepiece was split. Kaiser $(1872$ a,b) used the Airy micrometer to make two series of measures.

Birefringent prisms of the Muller, Rochon, and Wollaston types have been used to create another group of double-image micrometers. In the Rochon and Wollaston varieties, double images are produced whose separation is proportional to the distance of the prism from the focal plane, with the separation varying very slowly with the translation of the prism. This feature eliminates the need for a precision screw. However, the movement of the prism out of the focal plane to' obtain larger separations means increased aberrations. Muller $(1937,1939)$ showed that by reorienting the prisms, variation in separation could be accomplished by motion perpendicular to the optical axis, thus keeping aberrations to a minimum. This type of double-image micrometer remains the most successful, although its use today appears very limited.

Visual interferometry has also yielded important data, largely in the hands of W.S. Finsen $(1951,1954)$. He designed and built a clever eyepiece interferometer with movable slits, with which he made thousands of measures of close pairs at Johannesburg. His observational work also included an interferometer survey of the bright stars accessible to him, which yielded a number of new, fast moving binaries. Aside from those of Finsen, only small numbers of visual interferometer measures have been made, principally by Jeffers and Wilson with Anderson-type instruments, and by Danjon (1936) with a "half-wave interferential" device. By its very nature, visual interferometry is a great light waster, and that is undoubtedly its greatest drawback. Finsen could only observe pairs brighter than magnitude 7.5 with the 26 -inch refractor, while van den Bos was able to measure equally close pairs down to magnitude 9.5 or even fainter 
with the same instrument and the filar micrometer.

Despite the often ingenious instruments just described, the filar micrometer continues to maintain its lead as the most popular and productive instrument for visual double star measurement. Why is this so? I think that the answer is simply that the filar micrometer possesses a simplicity and versatility not equalled overall by its competitors. It can measure a wide range of separations acurately, is conservative of light, and is a speedy instrument to use. Furthermore, despite some claims to the contrary, its accuracy in the hands of a skilled observer is comparable to that attained by other devices. Nearly two decades ago, the micrometers employed at the Naval Observatory were provided with automatic readout and recording; this has improved their efficiency markedly. A complete measure, including all pertinent remarks, need take no longer than five minutes under good conditions.

\section{The Observers}

The 323000 means tabulated in Table 1 represent approximately 817000 measures made with filar micrometers. Nearly 600 observers have contributed this number, but the significant fact is that the "top" fifteen observers have made more than half of the total measures. These observers are listed in Table 2 , in order of decreasing numbers of measures. Values for Burnham are estimated,

Table 2.

Principal Visual Observers

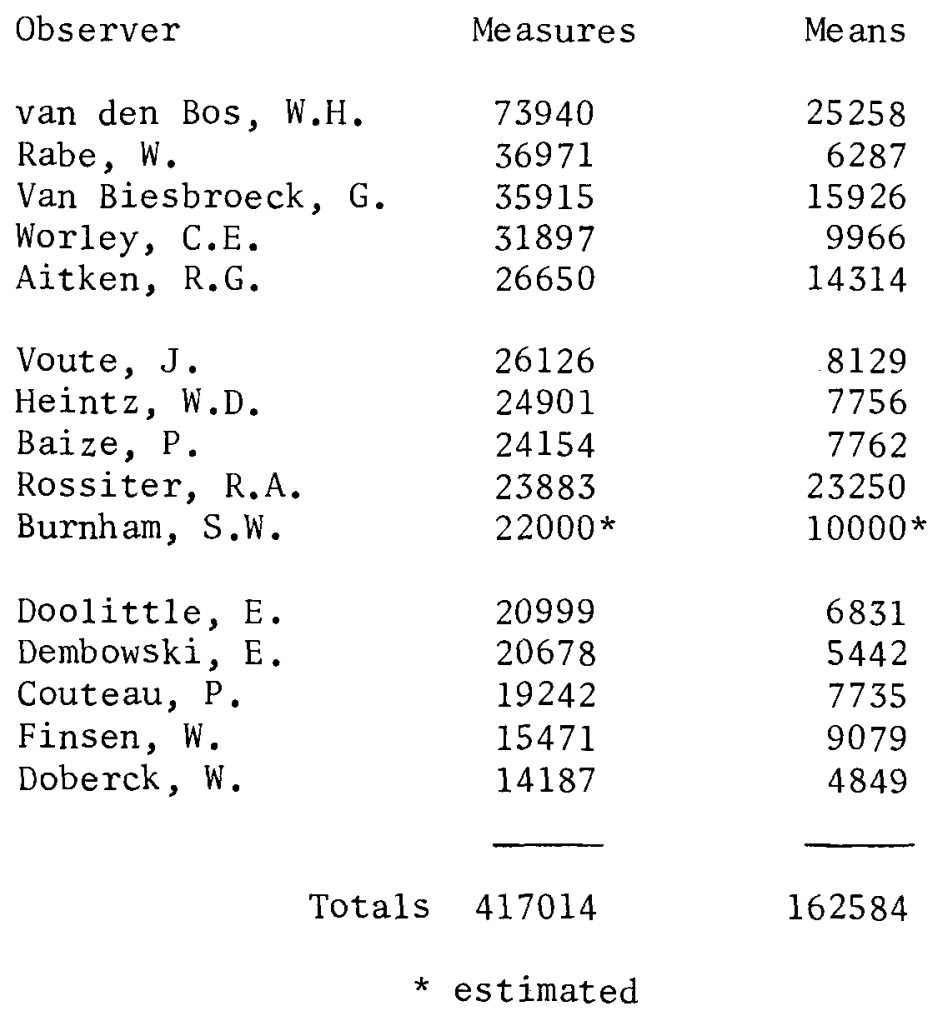


because, although all his observations have been punched, proofreading is not completed and the observations have not yet been added to the catalog. (Incidentally, of the observers who have made more than 10000 measures, only otto Struve is not yet punched: His total is estimated to be about 11000.) We see from Table 2 that the bulk of our knowledge concerning the orbital motions of visual binaries has come from a handful of observers, each of whom has worked for many years. Naturally, the quantity of observations is not the only criterion which must be used to evaluate the contribution of an individual observer. Certain of the observers in Table 2 used relatively small telescopes to make their measures, and consequently have less influence on the determination of orbits for the close pairs. But what is of most concern is the quality of the measures, for this ultimately determines the precision with which the mass-luminosity relation can be established.

How precisely can such measures be made? Table 3 lists three double stars of increasing magnitude difference, each of which has a definitive orbit which continues to represent the motion well. The interval studied is $1960-$ 1980 , during which each of these pairs revolved through more than two quadrants, with a variation in separation by a factor of two or more. The observers chosen are all experienced observers using telescopes of moderate aperture, and most of the observations were made with filar micrometers. Average residuals were formed for each observer, and are tabulated together with the number of means, nights, and the telescopes used. In evaluating these data, one caution is necessary: while my own observations cover the interval rather uniformly, those of the other observers in general do not. Hence, any errors related to changing separation may appear more prominent in one series than another. Note that, for all observers, the average residual in angle is around 0!:02, while that in separation is higher, perhaps 0!03 - 0!04. This confirms the well-known fact that angles can be measured visually with more precision than distances. Remembering that the average resolving power of the telescopes used is about $0 ! 2$, it appears that experienced visual observers achieve a precision of about $10 \%$ of this value in angle, and perhaps $15 \%$ to $20 \%$ in separation, for relatively close pairs. Recalling that visual measurements of photographic plates are said to attain a precision of about $1 \%$ of the image diameter, it would appear that visual observers do significantly less well. However, such a comparison is probably not valid, for the visual observer is dealing with entirely different conditions, such as seeing, telescope motion, physical discomfort, and micrometer wires which are an appreciable fraction of the diameter of the images he is trying to measure.

Fears concerning systematic errors in visual measures, and therefore the determinateness of the mass-luminosity relation, have long been expressed. Following the example of Otto Struve, who devised elaborate experiments to evaluate and correct his own measures for systematic errors, other observers and orbit computers in the late 19 th and early 20 th centuries spent much time in such pursuits. The unsuccessful aim of all this from our modern viewpoint, was to produce "corrected" data from which orbits could be computed. The fatal flaw was that the observed arcs were simply too short, and no amount of doctoring of the data could remedy this. Indeed, the perspicacious Agnes Clerke (1905) wrote:

"In no department of astronomy is the mischief of "personal equation" so sensible as in the measurement of double stars. Nearly all available data 
Tab1e 3 .

Precision of Visual Measures

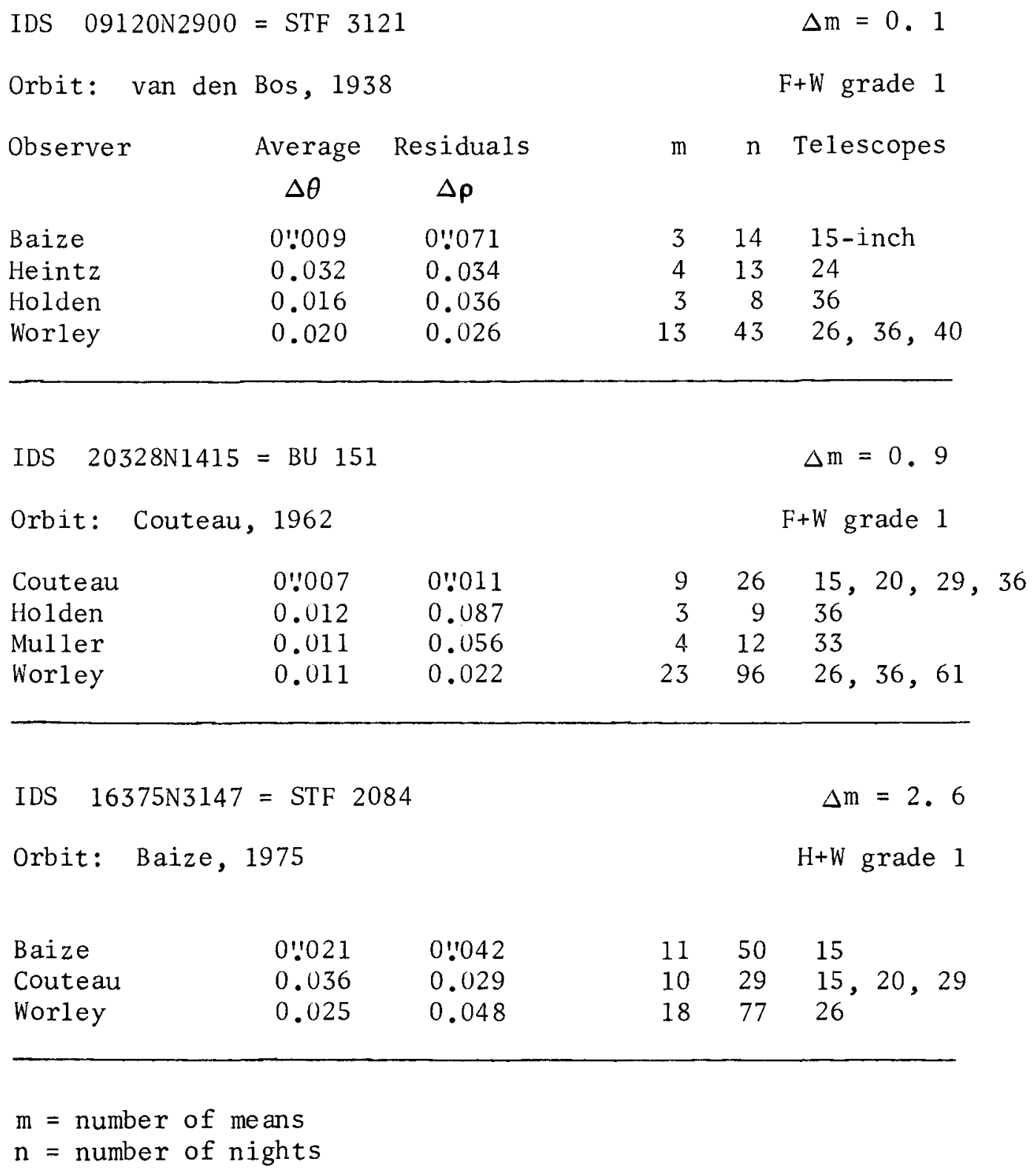


are prejudicially affected by it, and those emanating from different individual sources are thus often rendered exceedingly inharmonious. Much labor and ingenuity have been spent in determining its direction and amount for various observers, with a view to freeing their results from its effects; and, after all, it remains a question whether the observations so elaborately corrected are not more misleading than in their "raw" state."

Some years ago, in an attempt to evaluate this problem using modern data, G.G. Douglass and I (Worley and Douglass, 1970) examined over 14000 visual measures made by eleven experienced observers. The measures were referred to 51 definitive orbits, and residuals calculated. For four observers having long series of measures, we tested the dependence of the residuals on time, and found no obvious systematic effects. We then compared, observed, and computed separations for a1l eleven observers, and found small systematic effects in just four series. Finally, we detected a "proximity" effect, exhibited by all observers, when measuring the pairs close to or below the resolving power of their instruments. This effect caused the closest separations to be measured too large, while those slightly larger were measured too smal1. The entire range was smaller than $0: 1$, however. We concluded that there were no systematic effects large enough to seriously affect the mass-luminosity relation. Obviously, this study deserves a repeat when all of the measures are available in the observation Catalog. At that time, we plan such a study.

I note with interest that modern orbit computers pay little attention to deriving "personal equations", and that the orbits seem little the worse for it. On the other hand, inexperienced observers often show large accidental or systematic effects, which are impossible to disentangle in short series of measures, a fact well-known to most (but not all) orbit computers. Until the advent of speckle interferometry in the last half decade, no method existed for independently checking the reliability of visual measures of close pairs. Enough speckle measures have now accumulated from the work of McAlister and his associates (McAlister and Fekel, 1980) to permit such an evaluation, and the results are presented in Table 4 , where the first portion tabulates mean speckle residuals from orbits of high quality, and the second section presents

Table 4.

Comparison with Speckle Interferometry

Speckle residuals from visual orbits of high quality:

$$
\begin{aligned}
& \overline{\rho \Delta \theta}=+0.001 \pm 0.007 \\
& \overline{\Delta \rho}=-0.009 \pm 0.015 \quad(170 \text { obsns })
\end{aligned}
$$

Differences from nearly-simultaneous visual measures:

$$
\begin{aligned}
\overline{\Delta \theta} & =+0.34 \pm 3: 06 \\
\text { for } \quad \frac{\Delta \rho}{\Delta t} & =-0.021 \pm 0.042 \\
\text { and } & \frac{0.11 \pm 0.35 \mathrm{yr} .}{\rho}=0.38 \pm 0.19 \quad \text { (31 obsns) }
\end{aligned}
$$


a comparison of the speckle measures with nearly simultaneous visual measures made by the writer. It is encouraging to find that there is no discernable difference between the speckle results and the visual orbits, which in themselves represent a kind of "mean" or "standard" observer; nor does the comparison (admittedly less exact) between my measures and the speckle observations reveal any serious discrepancy. Thus, it appears that fears concerning systematic errors which might affect the mass-luminosity relation are groundless. Finally, I would encourage my visual colleagues to make a greater effort to provide contemporary observations of pairs known to be on the speckle observing lists, particularly in the next few years, in order to strengthen these conclusions.

\section{Orbits}

In the first edition of his book "The Binary Stars" Aitken (1918) listed 87 visual binaries with orbits. Since that time, the number of computed orbits has increased almost ten-fold, and there has been a steady increase in the quality. A new orbit catalog is in preparation by W.D. Heintz and the writer. Table 5 shows the present total number of orbits and their quality grades, as contrasted with the data available in the Finsen-Worley catalog (1970).

Table 5 .

Visual Orbits and Their Quality

Finsen-Wor ley

(1970)

Grade 1

2

3

4

5

Ambiguous
55

110

204

207

51

22

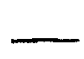

Total
Heintz-Worley

235

224

113

28

793

The grades are presently defined as follows:

Grade 1. Definitive. One revolution, or more, well observed. Grade 2. Reliable. One, or nearly one, revolution, well observed. Grade 3. Preliminary. Elements, (especially $P$ and a) not likely to be grossly in error. In general, the observations define at least half the orbit. Grade 4. Premature. Individual elements entitled to little weight, but $a^{\mathbf{3}} / \mathrm{P}^{\mathbf{2}}$ may be accepted with some confidence. In general, less than half the orbit is defined by observation.

Grade 5. Indeterminate. Observed arc very short with little curvature. 
Note that there are now nearly 200 orbits rated "Definitive" or "Reliable," and over 200 additional where the elements, particularly P and a, are likely to be reasonably accurate. Thus we now have 200-400 systems capable of yielding accurate masses. Recently, D. H. Popper (1980), in his review "tellar Masses" has remarked:

"It is rather sad, in view of the very great amount of difficult observing for more than 150 years, that the number of visual binaries for which masses are known to an accuracy of about $20 \%$ is not more than a dozen or so."

With this sentiment I heartily agree. The fault lies not with the visual observers, as we have seen, but with those who determine parallaxes and proper motions. Two problems exist. First, the "classical" photographic technique is not accurate enough to provide parallaxes which are precise enough for mass determinations beyond 20 parsecs. Second, parallax observers have been somewhat reluctant to give any priority to binaries, because these objects are more difficult to observe and interpret than single stars. Hopefully, the new parallax techniques now under development at several institutions will prove as accurate as their proponents anticipate. If so, visual double star observations, now beginning to be supplemented by impersonal techniques, have already provided a rich harvest of good and steadily improving orbits from which large numbers of accurate masses will result.

\section{References}

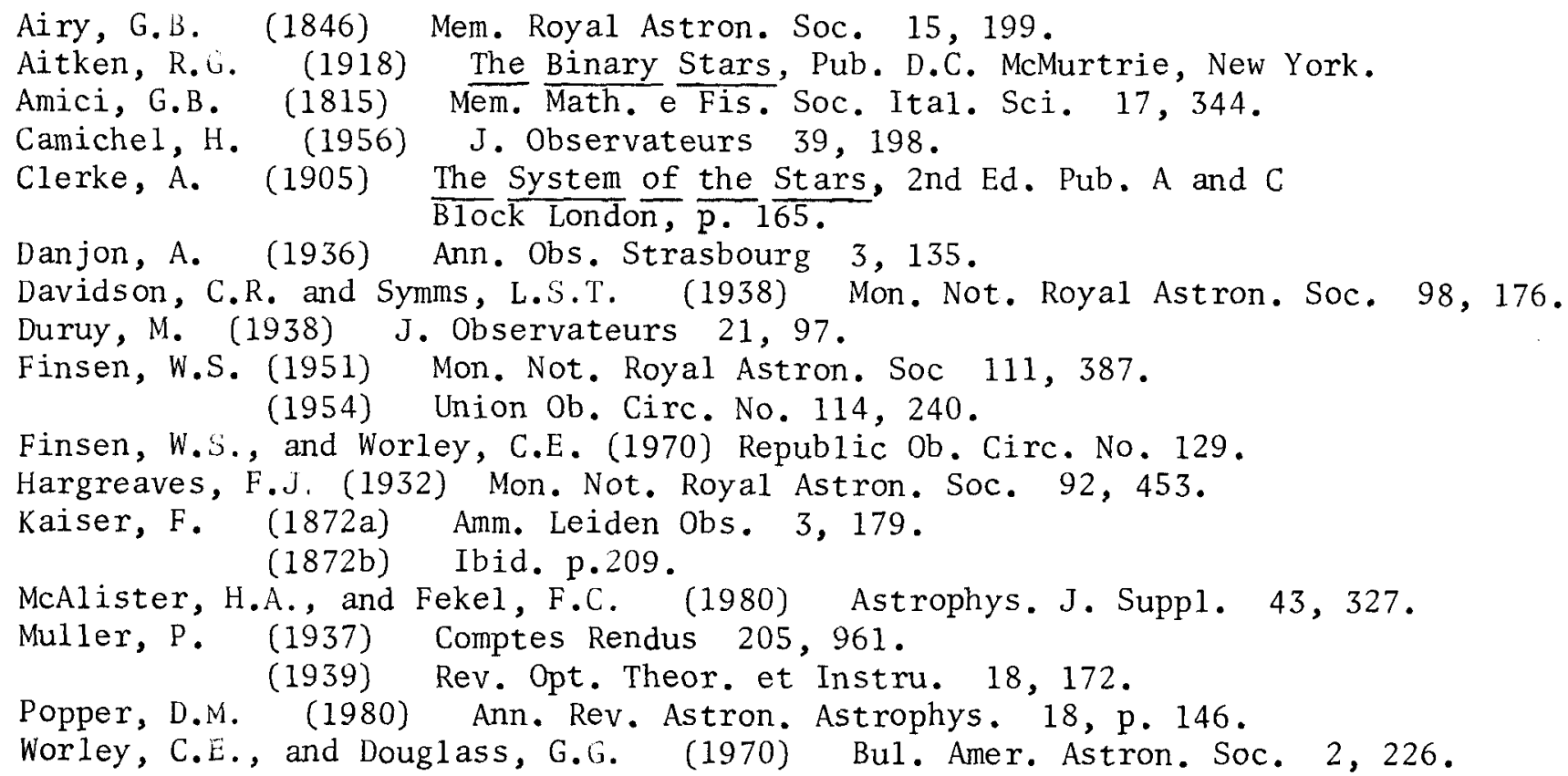




\section{DISCUSSION}

FREDRICK: Could you comment on the accuracy of the Muller double image micrometer measures?

WORLEY: There are several users of that instrument here in the audience. I have used it myself, mainly for the visual determination of magnitude differences. It has been touted as an instrument that is definitely superior, but I do not see that in the measures I have examined. There may be a slight advantage to it, but the objects that the observers have chosen are those with small magnitude differences and that are fairly bright, so one would expect better results anyway. The instrument has largely fallen into disuse. It is cumbersome to use, and I do not think it has met its promise of the past when it was thought to be such a superior instrument.

STRAND: You pointed out the need for improved parallaxes for the double stars with good orbits in order to get improved stellar masses. It should be emphasized that there is also a need for mass-ratio determinations, which require much longer time spans than parallax determinations, in order to determine individual masses.

WORLEY: Of course this is true, and I agree that it has to be emphasized for the parallax observers. On the other hand, there are a number of binaries where the magnitude differences are small and where, as has been done in the past in determining the mass-luminosity relation, we wimply assume equal masses for the two stars. This may not be correct, and we would like to have mass-ratio determinations in all cases.

POPPER: In a number of close visual systems, a very uncertain aspect is the determination of the color index or spectral type of the fainter component, which means the physical properties of that component may be poorly known. Without this knowledge, the interpretation of the system will not be possible. 